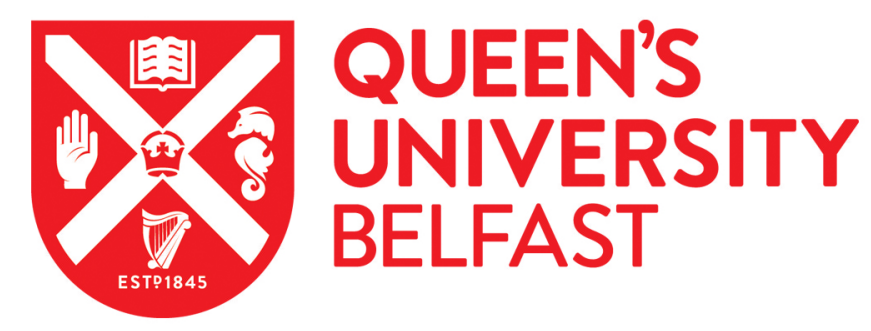

\title{
Acute respiratory distress syndrome subphenotypes and differential response to simvastatin: secondary analysis of a randomised
} controlled trial

Irish Critical Care Trials Group (2018). Acute respiratory distress syndrome subphenotypes and differential response to simvastatin: secondary analysis of a randomised controlled trial. The Lancet Respiratory Medicine, 6(9), 691-698. https://doi.org/10.1016/S2213-2600(18)30177-2

Published in:

The Lancet Respiratory Medicine

Document Version:

Peer reviewed version

Queen's University Belfast - Research Portal:

Link to publication record in Queen's University Belfast Research Portal

\author{
Publisher rights \\ Copyright 2018 Elsevier B. V. \\ This manuscript is distributed under a Creative Commons Attribution-NonCommercial-NoDerivs License \\ (https://creativecommons.org/licenses/by-nc-nd/4.0/), which permits distribution and reproduction for non-commercial purposes, provided the \\ author and source are cited.
}

\section{General rights}

Copyright for the publications made accessible via the Queen's University Belfast Research Portal is retained by the author(s) and / or other copyright owners and it is a condition of accessing these publications that users recognise and abide by the legal requirements associated with these rights.

\section{Take down policy}

The Research Portal is Queen's institutional repository that provides access to Queen's research output. Every effort has been made to ensure that content in the Research Portal does not infringe any person's rights, or applicable UK laws. If you discover content in the Research Portal that you believe breaches copyright or violates any law, please contact openaccess@qub.ac.uk. 


\section{ARDS Subphenotypes and Differential Response to Simvastatin: Secondary Analysis of a Randomized Controlled Trial}

Carolyn S. Calfee, MD;1,2,3 Professor Kevin L. Delucchi, PhD; Pratik Sinha, MD PhD; ${ }^{1}$ Professor Michael A. Matthay, MD;1,2,3 Jonathan Hackett, MB BCh; ${ }^{5}$ Manu Shankar-Hari PhD ${ }^{6,7}$; Cliona McDowell, MSc; ${ }^{8}$ Professor John G. Laffey, MD;9,10,11 Cecilia M. O'Kane, MB PhD ${ }^{5,12}$; Professor Daniel F. McAuley, MD ${ }^{5,12}$ and the Irish Critical Care Trials Group

\section{Affiliations:}

1 Department of Medicine, Division of Pulmonary, Critical Care, Allergy and Sleep Medicine; University of California, San Francisco; San Francisco, CA 2 Department of Anesthesia; University of California, San Francisco; San Francisco, CA 3 Cardiovascular Research Institute; University of California, San Francisco; San Francisco, CA 4 Department of Psychiatry; University of California, San Francisco; San Francisco, CA 5 Centre for Experimental Medicine, School of Medicine, Dentistry and Biomedical Sciences, Queen's University of Belfast, UK

6 Guy's and St Thomas' NHS Foundation Trust, ICU support Offices, $1^{\text {st }}$ Floor, East Wing, St Thomas' Hospital, SE1 7EH, UK 7 School of Immunology \& Microbial Sciences, Kings College London, SE1 9RT 8 Northern Ireland Clinical Trials Unit, The Royal Hospitals Belfast, BT12 6BA 9 Anaesthesia, School of Medicine, and Regenerative Medicine Institute (REMEDI) at CÚRAM Centre for Research in Medical Devices, National University of Ireland Galway, Galway, Ireland; 10 Keenan Research Centre for Biomedical Science, St. Michael's Hospital, Toronto;

11 Departments of Anesthesia, Physiology and Interdepartmental Division of Critical Care Medicine, University of Toronto, Toronto, Canada.

12 Regional Intensive Care Unit, The Royal Hospitals, Belfast BT12 6BA, UK.

Corresponding Author: Carolyn S. Calfee, MD MAS Associate Professor of Medicine, UCSF 505 Parnassus Ave, Box 0111 San Francisco, CA 94143-0111 Ph: 415/476-5756

Email: carolyn.calfee@ucsf.edu 


\section{ABSTRACT:}

Background: Precision medicine approaches targeting patients based on disease subtype have transformed approaches to cancer, asthma, and other heterogeneous syndromes. Two distinct subphenotypes of ARDS have been identified in three US-based clinical trials and respond differently to positive end-expiratory pressure and fluid management. It remains unknown if these subphenotypes exist in different populations and respond differently to pharmacotherapies.

Methods: We conducted a secondary analysis using data from 539 patients enrolled in a UK multicenter, placebo-controlled randomized trial of simvastatin for ARDS (HARP-2). Latent class analysis was applied to baseline data without consideration of outcomes to identify subphenotypes. Clinical outcomes were compared across subphenotypes and treatment groups.

Findings: A two class (two-subphenotype) model was an improvement over a one class model $(p<0.0001)$, with $65 \%$ of subjects in the hypo-inflammatory subphenotype and $35 \%$ in the hyperinflammatory subphenotype. Additional classes did not improve model fit. The clinical and biological characteristics of the two subphenotypes were similar to prior studies. While the original trial found no difference in 28-day survival between placebo and simvastatin, significantly different survival was identified across patients stratified by treatment and subphenotype $(p<0.0001)$. Specifically, within the hyper-inflammatory subphenotype, patients treated with simvastatin had significantly higher 28-day survival compared to placebo $(p=$ 0.008). A similar pattern was observed for 90-day survival.

Interpretation: Two subphenotypes of ARDS were identified in the HARP-2 cohort, with distinct clinical and biological features and disparate clinical outcomes; the hyper-inflammatory subphenotype had improved survival with simvastatin compared to placebo. These findings support further pursuit of predictive enrichment strategies in critical care clinical trials.

Word count: $257 / 250$ 


\section{RESEARCH IN CONTEXT}

Evidence before this study: Previous studies of ARDS patients, using data from U.S.-based randomized controlled trials, have identified two distinct subphenotypes with differential responses to mechanical ventilation and fluid therapy. It is unknown whether these subphenotypes can be identified in different populations using different datasets and, more importantly, whether these subphenotypes respond differently to pharmacotherapies. We searched PubMed on March 7, 2018 using the terms (ARDS or "acute lung injury") AND (subtype OR subphenotype OR endotype) and no language restrictions and identified no prior studies reporting differential responses to pharmacotherapy by ARDS subphenotype.

Added value of this study: This study reports that two distinct ARDS subphenotypes were identified in a secondary analysis of a U.K.-based randomized controlled trial of simvastatin for ARDS. Notably, the hyper-inflammatory subphenotype of ARDS had a survival benefit from simvastatin. To our knowledge, this study is the first to report differential response to pharmacotherapy by molecular subphenotype in ARDS.

Implications of all the available evidence: While other areas of medicine (e.g. cancer, asthma) have made significant progress by identifying biologically distinct subtypes of disease with differential treatment responses, critical care medicine has lagged behind. These findings suggest that targeting specific biological subtypes of critical illness syndromes in clinical trials may yield progress after decades of negative pharmacotherapy trials in the ICU. 


\section{INTRODUCTION}

The acute respiratory distress syndrome (ARDS) is a common and frequently fatal cause of respiratory failure among critically ill patients, with an incidence of nearly 200,000 cases per year in the US alone, an estimated prevalence of $10 \%$ among critically ill patients worldwide, and mortality of $30-40 \%,{ }^{1,2}$ ARDS is defined by clinical criteria including acute onset of hypoxemia $(\mathrm{PaO} 2 / \mathrm{FiO} 2$ ratio $<300 \mathrm{~mm} \mathrm{Hg}$ ), bilateral chest radiographic opacities, and exclusion of cardiac failure as the sole cause of the syndrome. ${ }^{3}$ Since the first consensus definition of ARDS in 1988, experts have debated whether patients should be subdivided by natural history, clinical features, biology, or some combination thereof. ${ }^{4}$ During the ensuing 3 decades, positive trials of several supportive care interventions, including most notably lung protective ventilation, have led to decreases in ARDS mortality - yet over the same time period, dozens of pharmacotherapies that seemed to show great promise in pre-clinical studies have failed in clinical trials. One of the often-cited reasons for this discouraging failure rate has been the considerable clinical and biological heterogeneity within ARDS, but objective data have been lacking to guide a more precision approach to clinical trials.

Latent class analysis is a well-validated statistical method that uses objective criteria to identify subgroups within a broader population. We previously applied latent class analysis in independent analyses of 3 cohorts of patients derived from NHLBI ARDS Network randomized controlled trials; in all 3 cohorts, summing to over 2000 patients, we observed strong evidence for two distinct and consistent subphenotypes of ARDS.5,6 In all 3 cohorts, one subphenotype representing roughly $30 \%$ of ARDS patients was consistently characterized by higher levels of inflammatory biomarkers, more profound shock and acidosis, and significantly worse clinical outcomes. Of particular interest, we found that this hyper-inflammatory subphenotype had a significantly different response to randomly assigned positive end-expiratory pressure (PEEP) and fluid management strategy, compared to the hypo-inflammatory subphenotype. ${ }^{5,6}$ These findings have suggested that improved understanding of these subphenotypes may be critical to 
future success in ARDS clinical trials. ${ }^{7}$ It remains unknown, however, whether these ARDS subphenotypes are generalizable to non-U.S. populations, whether they can be identified using less extensive datasets, and most importantly, whether they may respond differently to pharmacotherapies.

To test these questions, we designed a secondary analysis of a Phase 2B randomized controlled trial of simvastatin for ARDS, the Hydroxymethylglutaryl-CoA Reductase Inhibition with Simvastatin in Acute Lung Injury to Reduce Pulmonary Dysfunction-2 (HARP-2) Study. ${ }^{8}$ Based on our prior research, we hypothesized a priori that latent class analysis of the HARP-2 cohort would identify two distinct subphenotypes of ARDS with similar clinical and biological characteristics to those we have previously identified. We also hypothesized, based on the antiinflammatory effects of statins in laboratory and pre-clinical models of ARDS, ${ }^{9}$ that patients with the hyper-inflammatory subphenotype would preferentially respond to simvastatin.

\section{METHODS}

\section{Patient Sample}

HARP-2 was a multicenter randomized controlled trial conducted in the United Kingdom and Ireland comparing daily simvastatin $80 \mathrm{mg}$ to placebo in 540 patients with ARDS. ${ }^{8}$ One patient withdrew consent for the use of their data, so 539 patients were analyzed. Patients were enrolled within 48 hours of meeting ARDS criteria, and study drug was continued until Day 28, discharge from ICU, death, or development of a contraindication to continued statin therapy. The primary outcome of the study was ventilator-free days, with secondary outcomes of nonpulmonary organ failure free days and mortality; there were no significant differences in any of these outcomes by treatment allocation.

\section{Assay Procedures}


Interleukin 6 (IL-6) and soluble tumor necrosis factor receptor-1 (sTNFr-1) were measured for this analysis using plasma drawn prior to randomization and stored at $-80^{\circ} \mathrm{C}$. Biomarkers were measured in duplicate using commercially available enzyme-linked immunoassays (R\&D Systems).

\section{Statistical Analysis}

To estimate the optimal number of classes in the data, latent class models were fit in Mplus v8 ${ }^{10}$ using baseline demographics (age, gender), available baseline clinical data (direct and indirect ARDS risk factors, bilirubin, creatinine, platelet count, $\mathrm{PaO} 2 / \mathrm{FiO} 2$ ratio, plateau pressure, tidal volume, use of vasopressors), and baseline IL-6 and sTNFr1 as class-defining variables (Supplemental Table 1). Of note, fewer clinical and biomarker variables were available for these analyses than in our prior studies. Outcome variables were not included in the modeling. Models ranging from 1 to 4 classes were estimated to identify the optimal number of classes. From these four models, best-fit was evaluated using Bayesian Information Criteria (BIC), the Vuong-Lo-Mendell-Rubin likelihood ratio (VLMR) test (which compares fit of model $k$ classes to k-1 classes), class size, and entropy. ${ }^{11,12}$ Prior to beginning this modeling, variables were examined for their distribution, and continuous variables with significantly skewed distributions were log-transformed. To estimate model parameters, continuous variables were placed on a zscale with a mean of 0 and standard deviation of 1 , as in our prior work. ${ }^{5,6}$ As a sensitivity analysis, we repeated these models including C-reactive protein (CRP) levels. Additional details on the LCA modeling are in the supplementary appendix.

Once the optimal number of classes was determined, study participants were assigned to their most likely class, and their baseline characteristics were compared using t-tests, Pearson's chisquare, or Wilcoxon rank sum test depending on the nature of the variable. Associations between class assignment and clinical outcomes were tested using chi-square for mortality and 
Wilcoxon ranksum for ventilator-free days. To test for interactions between treatment and class assignment, we used logistic regression for mortality and zero-inflated Poisson regression for ventilator-free days. To test for differential response to treatment by class for survival, we compared time-to-event Kaplan-Meier curves using the log-rank test. For modeling time to unassisted breathing, a competing risks model was estimated with death before Day 28 as the competing risk. ${ }^{13}$ Analyses other than LCA were carried out using SAS (Version 9.4, (SAS Institute, Cary, NC, USA). Some results were previously reported in the form of an abstract. ${ }^{14}$

Role of the Funding Source: None

\section{Results}

\section{Population Characteristics}

Baseline characteristics of patients enrolled in the HARP-2 trial are fully described in the original publication and are summarized in Supplemental Table 2. Pneumonia was the most common contributing risk factor for ARDS (55\%). Overall, median ventilator-free days were 13 , and 28 -day mortality was $24.5 \%$.

\section{Two-class Model Optimally Fits the Population}

Analysis of the four LCA models showed that the 2-class model was a better fit for the population than a 1-class model, and additional classes did not improve model fit (Supplementary Table 3). Entropy in all models was 0.75 or greater, indicating adequate class separation. The Bayesian Information Criteria decreased as the number of classes in the model increased, indicating improved model fit with additional classes. The 2-class model had a significantly improved fit compared to the one-class model (VLMR $p<0.0001$ ); additional classes did not lead to significant improvement in model fit. These findings, in conjunction with the relatively small number of patients in the additional class in a 3-class model $(n=40)$, led us 
to proceed using a 2-class model. In the 2-class model, $353(65 \%)$ patients were assigned to class 1 and $186(35 \%)$ patients to class 2 . Of note, these proportions are consistent with our prior latent class analyses, ${ }^{5,6}$ despite the fact that the HARP-2 analyses used far fewer clinical and biomarker variables $(n=14)$ as inputs than the prior work $(n=35-37)$. Inclusion of CRP levels in the LCA models as a sensitivity analysis did not meaningfully affect the results (data not shown).

Average latent class probabilities were 0.93 for Class 1 and 0.92 for Class 2. These findings are also consistent with previous studies and indicative of robust class assignment.

\section{Comparison of Phenotypic Features and Outcomes Between Subphenotypes}

Class 2 had clinical and biological features similar to those found in prior studies and consistent with a hyper-inflammatory phenotype. Specifically, as compared to Class 1, patients in Class 2 had higher values of sTNFr-1 and IL-6, lower platelet counts (Figure 1; Table 1), and more vasopressor use (Table 1; $p<0.001)$. For simplicity, the classes will be referred to as the hypo-inflammatory subphenotype (Class 1 ) and the hyper-inflammatory subphenotype (Class 2 ) for the remainder of the manuscript. Age and gender were similar across the subphenotypes. Although the distribution of direct and indirect ARDS risk factors was significantly different across the two subphenotypes (Table 1; $p<0.0001$ ), the most common ARDS risk factors of sepsis, pneumonia and aspiration were highly prevalent among both groups, as in prior studies. Also similar to prior studies, the hyper-inflammatory subphenotype patients had fewer ventilatorfree days (median 2 vs 18 days; $p<0.0001$ ), fewer non-pulmonary organ failure free days (median 15 vs 27 days; $p<0.0001)$, and higher 28-day mortality ( $39 \%$ vs $17 \%, p<0.0001)$ compared to the hypo-inflammatory subphenotype (Table 2).

\section{Survival Benefit Observed with Simvastatin in Hyper-inflammatory Subphenotype}


The original trial found no difference in 28-day survival curves between placebo and simvastatin $(p=0.20)$. In contrast, we observed significantly different survival curves across patients stratified by treatment and subphenotype (Figure 2A; $p<0.0001)$. Specifically, within the hyperinflammatory subphenotype, patients treated with simvastatin had significantly higher 28-day survival compared to patients treated with placebo $(p=0.008)$. This effect was not observed in the hypo-inflammatory subphenotype patients. A similar pattern was observed for 90-day survival (Figure 2B; $p<0.0001$ for overall comparison; $p=0.03$ for hyper-inflammatory subphenotype simvastatin vs placebo).

In contrast to the curves stratified by subphenotype and treatment, survival curves stratified by ARDS severity (PF ratio) and treatment were not significantly different $(p=0.12)$. Survival curves stratified by APACHE II score (dichotomized at the mean) and treatment revealed no differential effect of treatment in either the high or low APACHE groups (Figure S1).

Mortality at 28 days was $32 \%$ in the hyper-inflammatory subphenotype patients treated with simvastatin, in comparison to $45 \%$ in the hyper-inflammatory subphenotype patients treated with placebo; in contrast, 28-day mortality was similar in patients in the hypo-inflammatory subphenotype regardless of treatment assignment (16\% vs $17 \%)$. Analysis of interaction between treatment and subphenotype for mortality was not statistically significant $(p=0.14)$.

In the original trial, time to unassisted breathing did not differ significantly between simvastatin and placebo patients. When stratified by subphenotype and treatment, time to unassisted breathing differed significantly (Figure 3; $p<0.0001$ ). However, the difference in the curves between the hyper-inflammatory subphenotype patients treated with simvastatin and placebo was not statistically significant $(p=0.10)$. In the hyper-inflammatory subphenotype, median ventilator-free days (VFD) were numerically higher in the simvastatin-treated patients compared 
to placebo (7 VFD vs 0 VFD), in contrast to patients in the hypo-inflammatory subphenotype where the median number of ventilator-free days was the same regardless of treatment (18 VFD in each); however, the interaction between treatment and subphenotype in regression models was not statistically significant $(p=0.15)$.

\section{DISCUSSION}

These analyses have two novel findings with important implications for future clinical trials in ARDS. First, two distinct ARDS subphenotypes with features similar to those we have previously reported were identified for the first time in a non-United States patient population and using a different and much smaller set of clinical and biomarker data than in previous studies. These findings indicate that these subphenotypes are consistent across geographic sites and are robust to variations in specific data collected, enhancing the generalizability of previous studies. Second, and more importantly, these two subphenotypes of ARDS responded differently to randomly assigned simvastatin, with evidence of improved survival at both 28 and 90 days uniquely among patients with a "hyper-inflammatory" subphenotype of ARDS. These findings suggest that identification of ARDS subphenotypes may be fundamentally important in future ARDS clinical trials and, more broadly, that targeting distinct subphenotypes of critical illness syndromes may finally yield progress after decades of negative pharmacotherapy trials in the ICU.

The heterogeneous, clinically defined syndromes of sepsis and ARDS are thought of as "graveyards" for novel pharmacotherapies, despite their high prevalence and mortality, and yet critical care has lagged behind other fields in its development of precision biomarker-guided treatments. ${ }^{15}$ The concept of targeting specific biomarker-defined subgroups of heterogeneous syndromes, a variant of precision medicine, has fundamentally changed the approach to patient care in oncology, with examples ranging from estrogen receptor status in breast cancer to BRAF mutation status in melanoma and other malignancies. ${ }^{16}$ In other fields, such as asthma, 
recognition of the importance of distinct subphenotypes is critical to the design of new clinical trials and is beginning to impact patient care. ${ }^{17}$ Over the past several years, there has been increasing evidence of the biological and clinical heterogeneity in sepsis and ARDS, including our prior work showing subphenotype-specific responses to PEEP and fluid management strategy, but until now, there has not been evidence that biologically distinct subphenotypes have differential response to pharmacotherapy in ARDS.

The finding that patients with a hyper-inflammatory ARDS subphenotype preferentially responded to randomly assigned simvastatin has biologic plausibility based on the presumed mechanism of action of statins in ARDS. Statins reduce lung inflammation and injury in both animal models of ARDS and pre-clinical human experimental studies ${ }^{9}$ and have endothelialstabilizing properties as well. Thus, patients with a higher degree of systemic inflammation such as those in the hyper-inflammatory subphenotype would seem to be most likely to respond to this therapy.

In this analysis as in our prior studies on the same topic, we note that extra-pulmonary factors (such as creatinine, bilirubin, and platelet levels) seemed to contribute more to subphenotype identification than pulmonary-specific variables such as $\mathrm{P} / \mathrm{F}$ ratio and ventilator parameters. One potential explanation for these findings is that patients were enrolled into this trial (as all ARDS clinical trials) on the basis of specific pulmonary criteria (e.g. PF ratio), while other pulmonary criteria (e.g. tidal volume, plateau pressure) are determined at least in part by protocols designed for ARDS patients. Thus, it is not entirely surprising that these pulmonary criteria converge and therefore contribute less to identifying subgroups of patients than nonpulmonary criteria. At the same time, it is also possible that the biological differences between subphenotypes either drive or are driven by multi-system organ failure, which then contributes to poorer outcomes.

We observed a clinically significant but not statistically significant difference in VFDs for patients in the hyper-inflammatory subphenotype treated with simvastatin (median 7 VFDs) vs 
placebo (median 0 VFDs). These data stand in contrast to the findings of the survival analysis (Figure 2), in which survival was significantly better with simvastatin in the hyper-inflammatory subphenotype. Our interpretation of these results is that the VFD analyses may be underpowered and likely reflect a pattern of preferential benefit to simvastatin in the hyperinflammary subphenotype similar to that identified in survival analysis, though other interpretations are also possible. Nevertheless, these findings highlight some of the challenges in using VFDs as an outcome for clinical trials in ARDS.

When considering the results of any subgroup analysis in a clinical trial, several important issues (in addition to biologic plausibility) must be considered, including multiple hypothesis testing, "post hoc" analyses, and statistical power and methodology. ${ }^{18}$ In the present analysis, only one subgroup analysis was pursued: specifically, latent classes were sought using an unbiased, data-driven approach that has identified distinct ARDS subphenotypes in 3 prior studies. Thus, multiple hypothesis testing (aside from the hypothesis of the original clinical trial) should not be an issue. This analysis was not planned as part of the original trial design because the trial was designed before our group's first description of ARDS subphenotypes. ${ }^{19}$ However, given our previous findings that ARDS subphenotypes responded differently to randomly assigned interventions (PEEP, fluid conservative therapy) in 2 prior large clinical trials, we thought it was an important hypothesis to test in this analysis. As with many subgroup analyses, the original HARP2 trial was not powered for this analysis; we also note that despite a $13 \%$ absolute risk reduction for mortality in the hyper-inflammatory subphenotype with simvastatin vs. placebo, the statistical test for interaction in the analyses of 28-day mortality was not statistically significant $(p=0.14)$. In this case, given the biologic plausibility of a preferential response to statins in the hyper-inflammatory subphenotype, as well as our prior findings of differential treatment responses to other interventions, we thought it was appropriate to directly compare the survival curves for hyper-inflammatory patients treated with simvastatin vs. 
placebo. A prospective clinical trial of simvastatin targeting hyper-inflammatory ARDS patients, however, must be conducted prior to making any treatment recommendations for this group.

This study has several strengths, including the consistency of the LCA results compared to prior studies, the data-driven and unbiased nature of LCA for subgroup identification, the biologic plausibility of the results, and the setting within a randomized controlled trial, which allows stronger causal inference regarding treatment effects, as compared with observational studies. This study also has some limitations, most of which derive from its origin as a subgroup analysis and are detailed above. As in some other prior ARDS clinical trials, ${ }^{20,21}$ plateau pressure was missing in a substantial proportion of patients $(45 \%)$. While latent class models can include patients with missing data, more complete data on this variable may have been helpful. In addition, because of the nature of latent class models, we note that it is not possible to prove that the two subphenotypes identified in this cohort are "the same" as the two subphenotypes identified in our prior studies, although the similarity of the clinical and biological variables distinguishing the two groups in this work and prior studies provides strong evidence in support of this hypothesis. As in nearly all randomized controlled trials in ARDS, mortality was lower in the HARP2 cohort than in contemporary observational ARDS cohorts; ${ }^{2}$ additional studies of ARDS subphenotypes in less carefully selected patient populations are needed. Finally, we wish to emphasize again that patients in the original trial were not randomized on the basis of their ARDS subphenotype, so in addition to the aforementioned caveats regarding subgroup analyses, it remains possible that unmeasured confounders could be contributing to our findings. Prospective confirmation of simvastatin benefit in the hyper-inflammatory subphenotype in a randomized controlled trial will be necessary.

Moving forward, how might these findings be translated to future clinical trials in ARDS? As reported in prior studies, the hyper-inflammatory ARDS subphenotype can be accurately identified using as few as 3 variables (e.g. IL-8, sTNFr1, bicarbonate). ${ }^{5,6}$ The development of the capability to measure these biomarkers in real time will be critical to conducting precision 
clinical trials in this setting. More broadly, these results suggest that predictive enrichment approaches to critical care clinical trials should be strongly considered. Investigators studying sepsis have identified distinct subtypes within that heterogeneous syndrome, defined by differences in whole blood gene expression, though testing for differential responses to randomly assigned treatment has not yet been carried out. ${ }^{22,23}$ If similar patterns are identified in sepsis, it would suggest that clinical trials in syndromes known to encompass significant biological heterogeneity should consider targeting patients based on their underlying biology rather than on a less specific syndromic diagnosis.

In conclusion, this secondary analysis of the HARP-2 trial of simvastatin for ARDS identifies two distinct subphenotypes of ARDS, one of which had significantly improved survival with simvastatin therapy compared to placebo. Two distinct subphenotypes of ARDS have now been identified in four different randomized controlled trial cohorts, with differential responses to mechanical ventilation, fluid management strategy, and now pharmacotherapy. These findings support further pursuit of predictive enrichment strategies in critical care clinical trials. 
FUNDING: CSC (HL140026). MSH is supported by the National Institute for Health Research Clinician Scientist Award (CS-2016-16-011). The views expressed in this article are those of the authors and not necessarily those of the Medical Research Council (MRC), National Health Service, National Institute for Health Research (NIHR), or Department of Health.

Supported by the U.K. Efficacy and Mechanism Evaluation (EME) Programme, an MRC and NIHR partnership (08/99/08 and 16/33/01). The EME Programme is funded by the MRC and NIHR, with contributions from the Chief Scientist Office in Scotland, the National Institute for Social Care and Health Research in Wales, and the Health and Social Care (HSC) Research and Development Division, Public Health Agency for Northern Ireland; by a Health Research Award (HRA_POR-2010-131) from the Health Research Board (HRB), Dublin; and by additional funding from the HSC Research and Development Division, Public Health Agency for Northern Ireland, the Intensive Care Society of Ireland, and Revive.

ACKNOWLEDGMENTS: We thank all the patients and their legal representatives who participated in the trial, all the research nurses and the pharmacists in all the participating centers, and the medical and nursing staff in participating centers who cared for patients and collected data; the staff of the Northern Ireland Clinical Trials Unit for support in conducting the trial; the staff from the HRB Galway Clinical Research Facility, Galway, Ireland, for help in conducting the study in Ireland; the staff of the Intensive Care National Audit and Research Centre (ICNARC) for providing APACHE II data for study sites that participate in the ICNARC Case Mix Programme; the staff of the Northern Ireland Clinical Research Network and the NIHR Clinical Research Network for help with patient recruitment and data acquisition and the U.K. Intensive Care Foundation. 


\section{REFERENCES}

1. Rubenfeld GD, Caldwell E, Peabody E, et al. Incidence and outcomes of acute lung injury. N Engl J Med 2005; 353(16): 1685-93.

2. Bellani G, Laffey JG, Pham T, et al. Epidemiology, patterns of care, and mortality for patients with acute respiratory distress syndrome in intensive care units in 50 countries. JAMA 2016; 315(8): 788-800.

3. Ranieri VM, Rubenfeld GD, Thompson BT, et al. Acute respiratory distress syndrome: the Berlin Definition. JAMA 2012; 307(23): 2526-33.

4. Bernard GR, Artigas A, Brigham KL, et al. The American-European Consensus Conference on ARDS. Definitions, mechanisms, relevant outcomes, and clinical trial coordination. Am J Respir Crit Care Med 1994; 149(3 Pt 1): 818-24.

5. Calfee CS, Delucchi K, Parsons PE, Thompson BT, Ware LB, Matthay MA. Subphenotypes in acute respiratory distress syndrome: latent class analysis of data from two randomised controlled trials. The Lancet Respiratory medicine 2014; 2(8): 611-20.

6. Famous KR, Delucchi K, Ware LB, et al. Acute Respiratory Distress Syndrome Subphenotypes Respond Differently to Randomized Fluid Management Strategy. Am J Respir Crit Care Med 2017; 195(3): 331-8.

7. Thompson BT, Chambers RC, Liu KD. Acute Respiratory Distress Syndrome. N Engl J Med 2017; 377(6): 562-72.

8. McAuley DF, Laffey JG, O'Kane CM, et al. Simvastatin in the Acute Respiratory Distress Syndrome. New England Journal of Medicine 2014; 371(18): 1695-703.

9. Shyamsundar M, McKeown ST, O'Kane CM, et al. Simvastatin decreases lipopolysaccharide-induced pulmonary inflammation in healthy volunteers. Am J Respir Crit Care Med 2009; 179(12): 1107-14.

10. Muthen LK, Muthen BO. Mplus Users Guide. 8th edition ed. Los Angeles, CA; 2017.

11. Schwartz G. Estimating the dimension of a model. The Annals of Statistics 1978; 6: 461-

4.

12. Lo YT, Mendell NR, Rubin DB. Testing the number of components in a normal mixture. Biometrika 2001; 88: 767-78.

13. Austin PC, Lee DS, Fine JP. Introduction to the Analysis of Survival Data in the Presence of Competing Risks. Circulation 2016; 133(6): 601-9.

14. Calfee CS, Delucchi KR, Matthay MA, et al. Consistent ARDS Endotypes Are Identified Using Minimal Data From A United Kingdom Clinical Trial. American Thoracic Society International Conference; 2017; Washington, DC; 2017.

15. Matthay MA, McAuley DF, Ware LB. Clinical trials in acute respiratory distress syndrome: challenges and opportunities. The Lancet Respiratory medicine 2017; 5(6): 524-34. 16. Rugo HS, Olopade OI, DeMichele A, et al. Adaptive Randomization of VeliparibCarboplatin Treatment in Breast Cancer. N Engl J Med 2016; 375(1): 23-34.

17. Wenzel SE. Asthma phenotypes: the evolution from clinical to molecular approaches. Nature medicine 2012; 18(5): 716-25.

18. Pocock SJ, Assmann SE, Enos LE, Kasten LE. Subgroup analysis, covariate adjustment and baseline comparisons in clinical trial reporting: current practice and problems. Statistics in medicine 2002; 21(19): 2917-30.

19. McAuley DF, Laffey JG, O'Kane CM, et al. Hydroxymethylglutaryl-CoA reductase inhibition with simvastatin in acute lung injury to reduce pulmonary dysfunction (HARP-2) trial: study protocol for a randomized controlled trial. Trials 2012; 13: 170. 
20. The Acute Respiratory Distress Syndrome Network. Ventilation with lower tidal volumes as compared with traditional tidal volumes for acute lung injury and the acute respiratory distress syndrome. N Engl J Med 2000; 342(18): 1301-8.

21. The Acute Respiratory Distress Syndrome Network. Higher versus lower positive endexpiratory pressures in patients with the acute respiratory distress syndrome. N Engl J Med 2004; 351(4): 327-36.

22. Davenport EE, Burnham KL, Radhakrishnan J, et al. Genomic landscape of the individual host response and outcomes in sepsis: a prospective cohort study. The Lancet Respiratory medicine 2016.

23. Scicluna BP, van Vught LA, Zwinderman AH, et al. Classification of patients with sepsis according to blood genomic endotype: a prospective cohort study. The Lancet Respiratory medicine 2017; 5(10): 816-26. 


\section{Figure Legends}

Figure 1: Continuous Variables By Subphenotype. Differences in standardized values of each continuous variable by subphenotype in the HARP-2 Study. The variables are sorted on the basis of the degree of separation between the subphenotypes, from maximum positive separation on the left (ie, hyper-inflammatory subphenotype higher than hypo-inflammatory subphenotype) to maximum negative separation on the right (ie, hyper-inflammatory subphenotype lower than hypo-inflammatory subphenotype). The y-axis represents standardized variable values, in which all means are scaled to zero and SDs to one. A value of +1 for the standardized variable signifies that the mean value for a given subphenotype was one SD higher than the mean value in the cohort as a whole. Mean values are joined by lines to facilitate displaying subphenotype profiles. STNFr1= soluble tumor necrosis factor receptor-1. IL-6 = interleukin-6.

Figure 2: Kaplan-Meier survival curves to 28 days (Figure 3A) and 90 days (Figure 3B) for patients in HARP-2, stratified by ARDS subphenotype and treatment (simvastatin vs placebo). Comparison of curves using the log-rank test. Figure $3 A$ : overall $p<0.0001$; comparison of hyper-inflammatory subphenotype patients treated with simvastatin vs placebo $p=0.008$. Figure 3B: overall $p<0.0001$; comparison of hyper-inflammatory subphenotype patients treated with simvastatin vs placebo $p=0.03$.

Figure 3: Time to unassisted breathing over 28 days, stratified by subphenotype and treatment condition, from Fine-Gray competing risks model. Overall $p<0.0001$; comparison of hyperinflammatory subphenotype patients treated with simvastatin vs. placebo $p=0.10$. 
Table 1: Values of Key Subphenotype-Defining Variables At Baseline, Stratified by Subphenotype

\begin{tabular}{|c|c|c|c|}
\hline Baseline Characteristic & $\begin{array}{c}\text { Hypo-inflammatory } \\
\text { Subphenotype } \\
(n=353)\end{array}$ & $\begin{array}{c}\text { Hyper-inflammatory } \\
\text { Subphenotype } \\
(n=186)\end{array}$ & P-Value \\
\hline Age & $51 \pm 16$ & $60 \pm 15$ & $<.0001$ \\
\hline Gender, n (\%) & $157(45)$ & $75(40)$ & .32 \\
\hline $\begin{array}{l}\text { Direct ARDS Risk Factors, } \\
\mathrm{n}(\%)\end{array}$ & & & $<.0001$ \\
\hline Aspiration & $36(10)$ & $13(7)$ & \\
\hline Pneumonia & $214(61)$ & $81(43)$ & \\
\hline Trauma & $26(7)$ & $5(3)$ & \\
\hline Other & $24(7)$ & $4(2)$ & \\
\hline None & $52(15)$ & $84(45)$ & \\
\hline $\begin{array}{l}\text { Indirect ARDS Risk Factors, } \\
\mathrm{n}(\%)\end{array}$ & & & $<.0001$ \\
\hline Sepsis & $116(33)$ & $108(58)$ & \\
\hline Pancreatitis & $4(1)$ & $14(7)$ & \\
\hline Other & $19(5)$ & $14(7)$ & \\
\hline None & $213(61)$ & $51(27)$ & \\
\hline Vasopressor Use, n (\%) & $205(58)$ & $151(81)$ & $<.0001$ \\
\hline P/F Ratio, kPa & $17.6 \pm 8$ & $16.1 \pm 7$ & .02 \\
\hline Plateau pressure, $\mathrm{cm} \mathrm{H} 20$ & $24 \pm 6$ & $24 \pm 6$ & .87 \\
\hline Tidal volume, $\mathrm{ml} / \mathrm{kg}$ & $8.3 \pm 2.6$ & $7.9 \pm 2.6$ & .099 \\
\hline Platelet count, thousands & $216 \pm 119$ & $148 \pm 114$ & $<.0001$ \\
\hline $\begin{array}{l}\text { Bilirubin, umol/L, median } \\
\text { (IQR) }\end{array}$ & $9(6-16)$ & $19.5(11-36)$ & $<.0001$ \\
\hline Creatinine, umol/L & $76 \pm 42$ & $156 \pm 91$ & $<.0001$ \\
\hline C-reactive protein, $\mathrm{mg} / \mathrm{L}$ & $174 \pm 109$ & $208 \pm 110$ & .0008 \\
\hline IL-6, pg/ml, median (IQR) & $79(35-197)$ & $348(133-1355)$ & $<.0001$ \\
\hline $\begin{array}{l}\text { sTNFr1, pg/ml, median } \\
(\text { IQR) }\end{array}$ & $3511(2382-5008)$ & $11202(7810-16703)$ & $<.0001$ \\
\hline $\begin{array}{l}\text { Randomized to simvastatin, } \\
\mathrm{n}(\%)\end{array}$ & $174(49)$ & 85 (45) & .38 \\
\hline
\end{tabular}

Values represent mean \pm standard deviation, unless otherwise specified.

Abbreviations: IL-6 = interleukin 6 . sTNFr1 $=$ soluble tumor necrosis factor receptor 1 .

Statistical comparison by Pearson's chi-square, t-test, or Wilcoxon rank-sum as appropriate. 
Table 2: Clinical Outcomes By Subphenotype

\begin{tabular}{|l|l|l|l|}
\hline & $\begin{array}{l}\text { Hypo- } \\
\text { inflammatory } \\
\text { Subphenotype } \\
(\mathbf{n = 3 5 3 )}\end{array}$ & $\begin{array}{l}\text { Hyper- } \\
\text { inflammatory } \\
\text { Subphenotype } \\
(\mathbf{n = 1 8 6 )}\end{array}$ & -value \\
\hline 28 Day Mortality, n (\%) & $59(17 \%)$ & $73(39 \%)$ & $<0.0001$ \\
\hline 90 Day Mortality, n (\%) & $78(22 \%)$ & $87(46 \%)$ & $<0.0001$ \\
\hline $\begin{array}{l}\text { Ventilator-Free Days, median (25- } \\
75 \%)\end{array}$ & $18(0-23)$ & $2(0-17)$ & $<0.0001$ \\
\hline $\begin{array}{l}\text { Non-pulmonary organ failure-free } \\
\text { days, median (25-75\%) }\end{array}$ & $27(21-28)$ & $15(0-25)$ & $<0.0001$ \\
\hline
\end{tabular}

Ventilator-free days and non-pulmonary organ failure-free days measured to day 28. 